neuropathy, the published article states, "Plasma exchange, cyclophosphamide, and intravenous immune globulin are all beneficial. . . ." However, in my original discussion of this case, I mentioned treatment with plasma exchange only in reference to early reports describing treatment of multifocal motor neuropathy with plasma exchange and cyclophosphamide in combination. My original statement was as follows: "Initial treatment studies described beneficial effects of plasma exchange and cyclophosphamide. Prednisone, in contrast, is ineffective and may worsen the disease. Subsequent studies have demonstrated the beneficial effects of intravenous immunoglobulin in multifocal motor neuropathy and the favorable risk-tobenefit ratio of this therapy relative to that of cytotoxic therapy has made IVIg the initial treatment of choice in these patients." This statement was changed during the editorial process, and the change erroneously implied that treatment of multifocal motor neuropathy with plasma exchange is beneficial.

William J. Triggs, M.D.

McKnight Brain Institute

Gainesville, FL 32611

\title{
Two Doses of Azithromycin to Eliminate Trachoma in a Tanzanian Community
}

TO THE EDITOR: Single-dose azithromycin is the first-choice antibiotic for the treatment of trachoma. The World Health Organization (WHO) currently recommends annual mass azithromycin treatment for 3 years in communities in which the prevalence of the clinical sign "trachomatous inflammation-follicular" in children between 1 and 9 years of age is $10 \%$ or more.

We previously reported the effect of high-coverage, single-dose mass azithromycin treatment on ocularChlamydia trachomatis infection in Kahe Mpya, Tanzania. ${ }^{1}$ In all, $97.6 \%$ of residents were treated; the prevalence of ocular $C$. trachomatis fell from $9.5 \%$ at baseline to $0.1 \% 24$ months later. We subsequently carried out a second round of mass treatment at 24 months, examined residents at 42 and 60 months, and took conjunctival swabs for testing for $C$. trachomatis by means of a polymerase-chain-reaction assay at 60 months. Just as at 6,12 , and 18 months, at 42 months, persons with active disease ( 21 of the 821 residents examined at 42 months) were offered tetracycline eye ointment. Field, laboratory, and statistical methods have been described previously, ${ }^{1,2}$ although because we expected the prevalence of infection to be low after the two mass treatments, we combined aliquots from each of five eluted swabs for each assay, intending to retest individual samples if results were positive or equivocal. ${ }^{3}$ Approval for the study was obtained from ethics committees at the London School of Hygiene and Tropical Medicine and Tumaini University.

At 24 months (when the prevalence of infection was $0.1 \%^{1}$ ), the rate of antibiotic coverage was $93.1 \%$ (917 of 985 persons). At 42 months, 821 of 975 residents (84.2\%) were examined, as were 859 of 964 (89.1\%) at 60 months. The prevalence of trachomatous inflammation-follicular in children between 1 and 9 years of age fell from $16.3 \%$ at 24 months to $4.6 \%$ at 42 months and $2.6 \%$ at 60 months. At 60 months (3 years after the second mass treatment), $C$. trachomatis DNA was not detected in the conjunctiva of any of the 859 residents from whom swab specimens were obtained, suggesting that infection may have been eliminated.

One or two rounds of high-coverage mass treatment with azithromycin may be sufficient to eliminate ocular $C$. trachomatis in communities with moderate levels of infection. In this Tanzanian community, the fall in the prevalence of trachomatous inflammation-follicular lagged considerably behind the fall in the prevalence of infection (Fig. 1). Had WHO recommendations on antibiotic use been followed, three or possibly five annual rounds of mass treatment would have been offered, whereas our data suggest that one round was sufficient. A field-based assay for estimating the prevalence of infection ${ }^{4}$ is needed to guide treatment decisions in the 3 to 5 years after the first distribution of antibiotics for trachoma control.

\footnotetext{
Anthony W. Solomon, Ph.D.

Emma Harding-Esch, M.Sc.

Neal D.E. Alexander, Ph.D.

Aura Aguirre, Ph.D.

Martin J. Holland, Ph.D.
} 


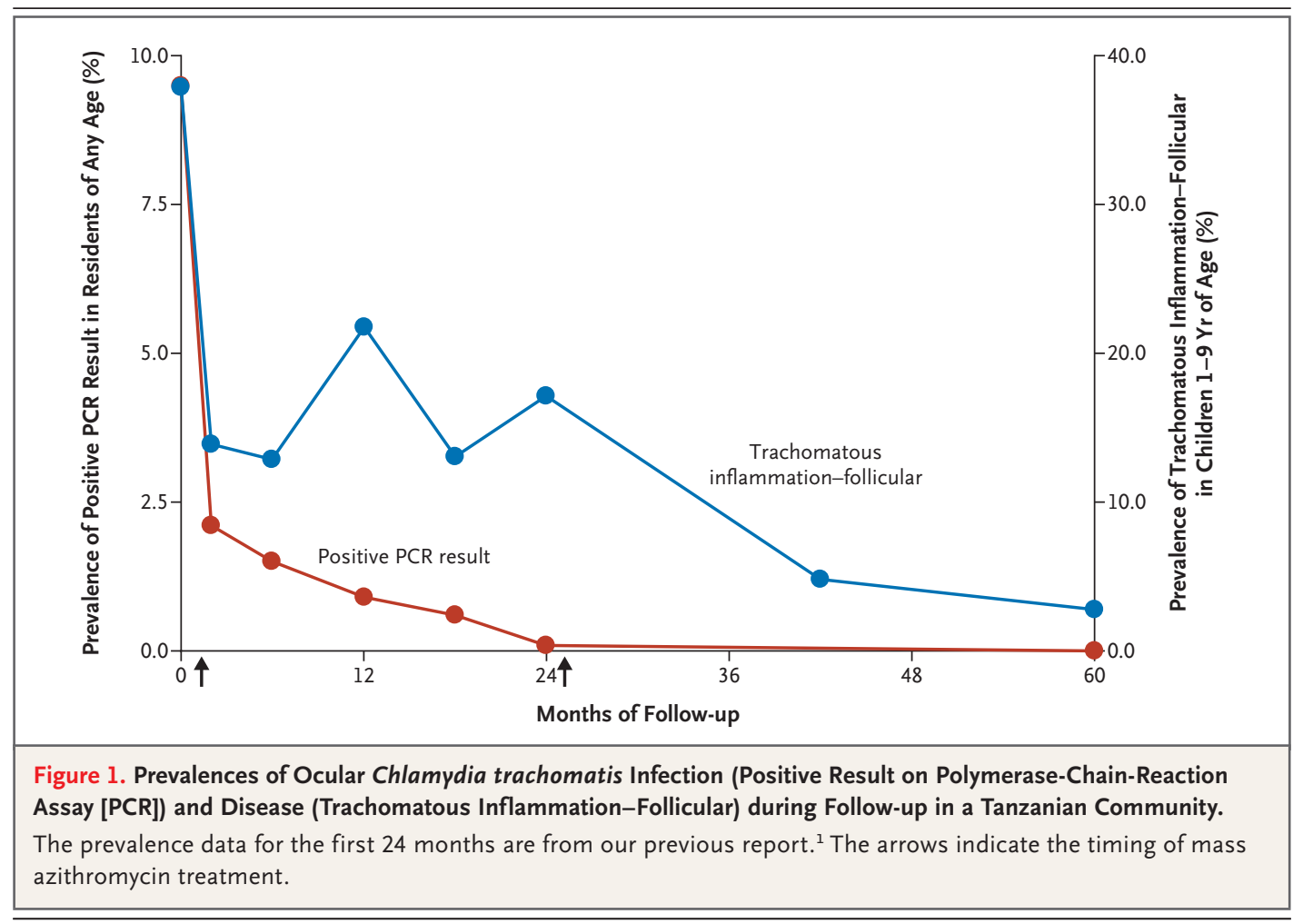

Robin L. Bailey, Ph.D.

Allen Foster, F.R.C.S., F.R.C.Ophth. David C.W. Mabey, D.M.

London School of Hygiene and Tropical Medicine

London WCIE 7HT, United Kingdom

anthony.solomon@lshtm.ac.uk

Patrick A. Massae, D.C.E.H.

Huruma Hospital

Rombo, Tanzania

Paul Courtright, Dr.P.H.

John F. Shao, M.D., Ph.D.

Tumaini University

Moshi, Tanzania

Supported by grants from Pfizer (2005-0812) and the Wellcome Trust-Burroughs Wellcome Fund (059134).
Drs. Solomon, Bailey, and Mabey report receiving grant support from the International Trachoma Initiative, which is partly funded by Pfizer, the manufacturers of azithromycin; and Drs. Solomon and Mabey, support from Pfizer to attend an international meeting on trachoma research. No other potential conflict of interest relevant to this letter was reported.

1. Solomon AW, Holland MJ, Alexander ND, et al. Mass treatment with single-dose azithromycin for trachoma. N Engl J Med 2004;351:1962-71.

2. Solomon AW, Holland MJ, Burton MJ, et al. Strategies for control of trachoma: observational study with quantitative PCR. Lancet 2003;362:198-204.

3. Diamant J, Benis R, Schachter J, et al. Pooling of Chlamydia laboratory tests to determine the prevalence of ocular Chlamydia trachomatis infection. Ophthalmic Epidemiol 2001;8:109-17.

4. Michel CE, Solomon AW, Magbanua JP, et al. Field evaluation of a rapid point-of-care assay for targeting antibiotic treatment for trachoma control: a comparative study. Lancet 2006;367:1585-90. Correspondence Copyright @ 2008 Massachusetts Medical Society.

INSTRUCTIONS FOR LETTERS TO THE EDITOR

Letters to the Editor are considered for publication, subject to editing and abridgment, provided they do not contain material that has been submitted or published elsewhere. Please note the following: - Letters in reference to a Journal article must not exceed 175 words (excluding references) and must be received within 3 weeks after publication of the article. Letters not related to a Journal article must not exceed 400 words. All letters must be submitted over the Internet at http://authors.nejm.org. -A letter can have no more than five references and one figure or table. $\cdot$ A letter can be signed by no more than three authors. - Financial associations or other possible conflicts of interest must be disclosed. (Such disclosures will be published with the letters. For authors of Journal articles who are responding to letters, this information appears in the published articles.) -Include your full mailing address, telephone number, fax number, and e-mail address with your letter.

Our Web site: http://authors.nejm.org

We cannot acknowledge receipt of your letter, but we will notify you when we have made a decision about publication. Letters that do not adhere to these instructions will not be considered. Rejected letters and figures will not be returned. We are unable to provide prepublication proofs. Submission of a letter constitutes permission for the Massachusetts Medical Society, its licensees, and its assignees to use it in the Journal's various print and electronic publications and in collections, revisions, and any other form or medium. 